\title{
Offline and online detection of damage using Autoregressive models and Artificial Neural Networks
}

\author{
Piotr Omenzetter*, Oliver R. de Lautour \\ Department of Civil and Environmental Engineering, The University of Auckland, Private Bag \\ 92019, Auckland Mail Center, Auckland 1142, New Zealand
}

\begin{abstract}
Developed to study long, regularly sampled streams of data, time series analysis methods are being increasingly investigated for the use of Structural Health Monitoring. In this research, Autoregressive (AR) models are used in conjunction with Artificial Neural Networks (ANNs) for damage detection, localisation and severity assessment. In the first reported experimental exercise, AR models were used offline to fit the acceleration time histories of a 3-storey test structure in undamaged and various damaged states when excited by earthquake motion simulated on a shake table. Damage was introduced into the structure by replacing the columns with those of a thinner thickness. Analytical models of the structure in both damaged and undamaged states were also developed and updated using experimental data in order to determine structural stiffness. The coefficients of AR models were used as damage sensitive features and input into an ANN to build a relationship between them and the remaining structural stiffness. In the second, analytical exercise, a system with gradually progressing damage was numerically simulated and acceleration AR models with exogenous inputs were identified recursively. A trained ANN was then required to trace the structural stiffness online. The results for the offline and online approach showed the efficiency of using AR coefficient as damage sensitive features and good performance of the ANNs for damage detection, localization and quantification.
\end{abstract}

Keywords: Structural Health Monitoring, damage detection, Autoregressive models, time series analysis, Artificial Neural Networks, model updating

\section{INTRODUCTION}

Despite considerable research efforts that Structural Health Monitoring (SHM) has received over the past decades, a robust and reliable method capable of detecting, locating and quantifying damage whilst being insensitive to changes in environmental and operating conditions has yet to be agreed upon. It appears that vibration based SHM methods are the most promising. While extensive literature reviews of such methods can be found elsewhere ${ }^{1,2}$, the focus here is restricted to the problems and techniques relevant for this study, namely, the applications of time series methods, detection of seismic damage, and the use of Artificial Neural Networks (ANNs) for recognizing patterns in responses of healthy and damaged structures.

Time series techniques appear to be inherently suitable to SHM because they were developed to analyse long sequences of data sampled at regular intervals, which are also often obtained in SHM exercises. However, the application of these techniques to SHM has yet to be fully explored. Examples of studies where time series models form the basis for choosing damage sensitive features include Sohn et al. ${ }^{3}$ who used Autoregressive (AR) models to fit the dynamic response of a concrete bridge pier. By performing statistical analysis on the coefficients of the AR models the authors were able to distinguish between healthy and damaged systems. A later study by Sohn et al. ${ }^{4}$ applied a similar methodology to health monitoring of a surface-effect fast patrol boat. However, the authors did not attempt to locate or quantify damage. Recently, Omenzetter and Brownjohn ${ }^{5}$ used a vector Seasonal Autoregressive Integrated Moving Average model to detect abrupt changes in strain data collected from the continuous monitoring of the SingaporeMalaysia Second Link Bridge. The seasonal model was used because of the strong diurnal component in the data caused by temperature variations. Nair et al. ${ }^{6}$ used an Autoregressive Moving Average time series to model the vibration signal from the ASCE benchmark structure. The authors defined a damage sensitive feature used to discriminate between the damaged and undamaged states of the structure based on the first three AR coefficients. The localization of damage was

*p.omenzetter@auckland.ac.nz; phone 649 373-7599 ext. 88138; fax 64 9 373-7462; http://www.cee.auckland.ac.nz 
achieved by introducing another feature, also based on the AR coefficients, found to increase from a baseline value when damage was near. While the previous references were concerned with linear time series models, the use of non-linear time series for damage detection has also begun to attract attention ${ }^{7-9}$. For the classification of damage sensitive features various statistical ${ }^{3,10}$ and pattern recognition techniques, such ANNs, have been used. The applications of ANNs for seismic damage detection have mostly been restricted to frequency domain data ${ }^{11,12}$, although Nakamura et al. ${ }^{13}$ directly analysed time histories of seismic response of a building.

The contribution of this study is the development of a method for seismic structural damage detection that integrates the use of AR models to establish damage sensitive features and application of ANNs for identification of damage presence, location and severity.

Two damage detection approaches were considered: an offline and an online. The data used throughout the offline study were measured on a 3-storey, shear-type, laboratory structure mounted on a shake-table (Fig. 1). Several damage cases were introduced to the structure by replacing the columns with thinner ones and damage was considered to be a reduction in lateral stiffness. Concurrently, simple, analytical, proportionally damped mass-spring dynamic models for the structure were formulated for each damage state. The laboratory structure was excited by a suite of earthquake motions and accelerations at each floor were measured. Firstly, experimental modal analysis was performed on both the healthy and damaged structures and the identified modal parameters were used to update the computer models. From these models the lateral stiffness of each storey was estimated. The approach to damage detection was to fit the acceleration time histories from each storey of the structure in the undamaged and various damaged states with AR models. The coefficients of these AR models were chosen as damage sensitive features and used as inputs into an ANN. Two damage assessment exercises of increasing complexity were conducted. Initially, the ANN was trained to recognize changes in the patterns of the AR coefficients and generally classify damage into states. Secondly, the ANN was trained to quantify the damage at each storey in terms of remaining stiffness.

This paper also reports on preliminary investigations on an online damage detection approach. A simple analytical model of a system with gradually progressing damage, modelled as time varying stiffness, was considered. Accelerations of the system were modelled using an AR model with eXogenous inputs (ARX). Because of the time-varying stiffness, the ARX model was identified recursively using a Kalman filter. An ANN was again used to map the changes in the ARX model coefficients into the changes in structural stiffness, but this time was expected to trace the stiffness changes online. In both offline and the online case the ANNs were shown to be able to detect and classify or quantify the damage with good accuracy.

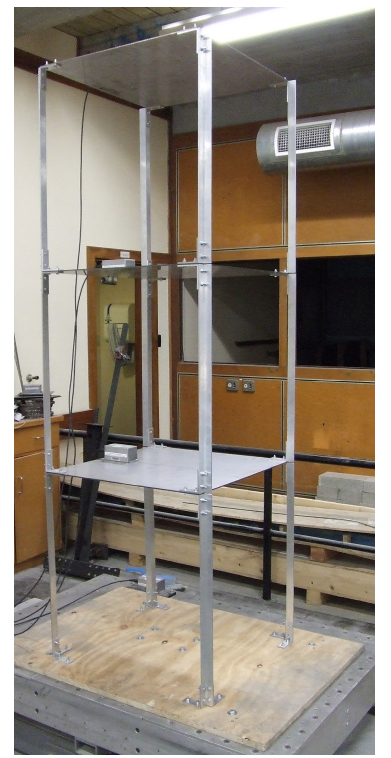

(a)

Fig. 1. Test structure: (a) general view, (b) detail of column-plate joint, and (c) dimensions and accelerometer locations.

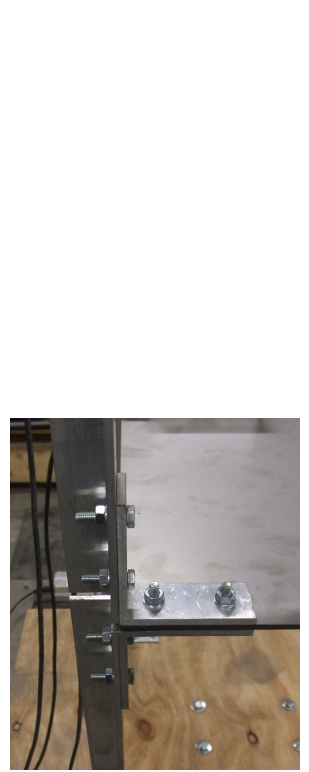

(b)

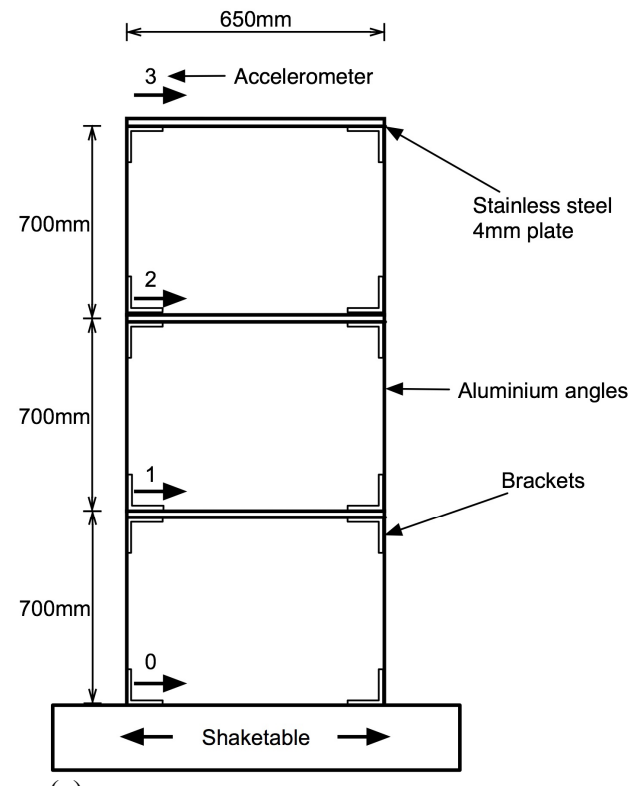

(c) 


\section{THEORY}

\section{Autoregressive models}

In this research, AR time series models are used to describe the acceleration time histories. AR models are a staple of time series analysis ${ }^{14}$ and are often used for modelling of stationary time series processes. A stationary process is a stochastic process, one that obeys probabilistic laws, in which the mean, variance and higher order moments are time invariant. AR models considered in this research are either output-only, i.e. pure AR formulations, or ARX models. AR models attempt to account for the correlations of the current observation in time series with its predecessors and also, in the case of $\mathrm{ARX}$ formulations, with known inputs. A univariate $\mathrm{ARX}$ model of order $(p, q)$, or $\mathrm{ARX}(p, q)$, can be written as:

$$
x_{t}=a_{1} x_{t-1}+a_{2} x_{t-2}+\ldots+a_{p} x_{t-p}+b_{0} u_{t-k}+b_{1} u_{t-k-1}+\ldots+b_{q} u_{t-k-q}+e_{t}
$$

where $x_{t}, \ldots x_{t-p}$ are the current and previous values of the output series $\left\{x_{t}\right\}(t=1,2, \ldots n), u_{t-k}, \ldots u_{t-k-q}$ are the values of the input series $\left\{u_{t}\right\}$ each shifted by a delay of $k$ time periods, and $\left\{a_{t}\right\}$ is a Gaussian white noise error time series with a zero mean. The ARX coefficients are denoted by $a_{1}, \ldots a_{p}, b_{1}, \ldots b_{p}$. Note that by assuming that all input coefficients in Eq. (1) are zero one obtains a pure $\operatorname{AR}(p)$ model. The ARX coefficients for time-invariant models can be evaluated using a variety of methods ${ }^{14}$. In this study, the coefficients were calculated using a least-squares solution. Given a series of $n$ observations, Eq. (1) can be rewritten into matrix form:

$$
\mathbf{e}=\mathbf{y}-\mathbf{X} \phi
$$

where

$$
\begin{aligned}
& \mathbf{e}=\left[\begin{array}{lll}
e_{n} & \ldots & e_{\max (p+1, k+q+1)}
\end{array}\right]^{T} \\
& \mathbf{y}=\left[\begin{array}{lll}
x_{n} & \ldots & x_{\max (p+1, k+q+1)}
\end{array}\right]^{T} \\
& \mathbf{X}=\left[\begin{array}{cccccc}
x_{n-1} & \ldots & x_{n-p} & u_{n-k} & \ldots & u_{n-k-q} \\
\vdots & \vdots & \vdots & \vdots & \vdots & \vdots \\
x_{\max (p+1, k+q+1)-1} & \ldots & x_{\max (p+1, k+q+1)-p} & u_{\max (p+1, k+q+1)-k} & \ldots & u_{\max (p+1, k+q+1)-k-q}
\end{array}\right] \\
& \phi=\left[\begin{array}{llllll}
a_{1} & \ldots & a_{p} & b_{0} & \ldots & b_{q}
\end{array}\right]^{T}
\end{aligned}
$$

In Eq. (2)-(6), $\mathbf{e}$ is an error vector, $\mathbf{y}$ is a vector containing the current outputs, $\mathbf{X}$ is a matrix of the previous outputs and inputs, and $\phi$ is a vector containing the $p+q$ ARX coefficients. Superscript $T$ denotes transpose. A least-squares solution seeks to minimize the sum of squared errors:

$$
\mathbf{e}^{T} \mathbf{e}=(\mathbf{y}-\mathbf{X} \phi)^{T}(\mathbf{y}-\mathbf{X} \boldsymbol{\phi})
$$

Hence by differentiation of Eq. (7) with respect to vector $\phi$ the ARX coefficients can be calculated from:

$$
\phi=\left(\mathbf{X}^{T} \mathbf{X}\right)^{-1} \mathbf{X}^{T} \mathbf{y}
$$

\section{Recursive identification using Kalman filter}

While the above least-squares scheme enables offline identification of time invariant AR models it does not explicitly address the problem of identification of systems that vary with time, e.g. as a result of gradually developing damage. Various recursive identification algorithm exist ${ }^{15}$, such as the forgetting factor or Prediction Error Method (PEM); in this study the popular Kalman filter is used ${ }^{16}$.

Consider the following state space model:

$$
\mathbf{y}_{t}=\mathbf{Z}_{t} \boldsymbol{\alpha}_{t}+\boldsymbol{\varepsilon}_{t}
$$




$$
\boldsymbol{\alpha}_{t}=\mathbf{T}_{t} \boldsymbol{\alpha}_{t-1}+\boldsymbol{\eta}_{t}
$$

Equation (9) is referred to as the measurement equation, and Eq. (10) as the transition equation, respectively, where $\mathbf{y}_{t}$ is the vector of outputs and $\boldsymbol{\alpha}_{t}$ is the vector of states. Matrices $\mathbf{Z}_{t}$ and $\mathbf{T}_{t}$ are measurement and transition matrices, respectively. Vectors $\varepsilon_{t}$ and $\eta_{t}$ represent multivariate zero mean Gaussian disturbances with covariance matrices $\mathbf{H}_{t}$ and $\mathbf{Q}_{t}$, respectively. These disturbances can also be contemporaneously correlated, such that

$$
E\left(\boldsymbol{\eta}_{t} \boldsymbol{\varepsilon}_{s}^{T}\right)=\left\{\begin{array}{cc}
\mathbf{G}_{t}, & t=s \\
\mathbf{0}, & t \neq s
\end{array}\right.
$$

where $E$ denotes the expected value operator.

The optimal estimation $\mathbf{a}_{t}$ of the state vector $\boldsymbol{\alpha}_{t}$ conditional on the information available at time $t$ can be obtained through recursive application of the following prediction equations:

$$
\begin{gathered}
\mathbf{a}_{t \mid t-1}=\mathbf{T}_{t} \mathbf{a}_{t-1} \\
\mathbf{P}_{t \mid t-1}=\mathbf{T}_{t} \mathbf{P}_{t-1} \mathbf{T}_{t}^{T}+\mathbf{Q}_{t}
\end{gathered}
$$

and updating equations:

$$
\begin{gathered}
\mathbf{a}_{t}=\mathbf{a}_{t \mid t-1}+\left(\mathbf{P}_{t \mid t-1} \mathbf{Z}_{t}^{T}+\mathbf{G}_{t}\right) \mathbf{F}_{t}^{-1}\left[\mathbf{y}_{t}-\mathbf{z}_{t}\left(\mathbf{a}_{t \mid t-1}\right)\right] \\
\mathbf{P}_{t}=\mathbf{P}_{t \mid t-1}-\left(\mathbf{P}_{t \mid t-1} \mathbf{Z}_{t}^{T}+\mathbf{G}_{t}\right) \mathbf{F}_{t}^{-1}\left(\mathbf{Z}_{t} \mathbf{P}_{t \mid t-1}^{T}+\mathbf{G}_{t}^{T}\right)
\end{gathered}
$$

with

$$
\mathbf{F}_{t}=\mathbf{Z}_{t} \mathbf{P}_{t \mid t-1} \mathbf{Z}_{t}^{T}+\mathbf{Z}_{t} \mathbf{G}_{t}+\mathbf{G}_{t}^{T} \mathbf{Z}_{t}^{T}+\mathbf{H}_{t}
$$

Matrix $\mathbf{P}_{t}$ is the covariance matrix of the estimation error:

$$
\mathbf{P}_{t}=E\left[\left(\boldsymbol{\alpha}_{t-1}-\mathbf{a}_{t \mid t-1}\right)\left(\boldsymbol{\alpha}_{t-1}-\mathbf{a}_{t \mid t-1}\right)^{T}\right]
$$

Taken together, Equations (12)-(16) form the Kalman filter.

For the purpose of identification of coefficients of the ARX model of Eq. (1), the output and state vectors were respectively defined as follows:

$$
\begin{aligned}
& \mathbf{y}_{t}=x_{t} \\
& \boldsymbol{\alpha}_{t}=\phi_{t}
\end{aligned}
$$

where $x_{t}$ and $\phi_{t}$ are defined in Eq. (1) and Eq., (6) respectively. In Eq. (19), superscript $t$ emphasizes that ARX coefficients can vary with time. The transition equation (Eq. (10)) was assumed in the following, so-called random walk form $^{14}$ :

$$
\boldsymbol{\alpha}_{t}=\boldsymbol{\alpha}_{t-1}+\boldsymbol{\eta}_{t}
$$

The rationale behind this choice was that under the null hypothesis the parameters of the system are assumed not to change with time, except for some stochastic uncertainty in their identification.

\section{Artificial Neural Networks}

To classify damage sensitive features of the signals, in this case AR coefficients, coming from the undamaged and damaged structure ANNs are used. ANNs are structures deliberately designed to utilize the organizational principles found in the brain. ANNs are capable of pattern recognition, classification and function approximation tasks and have been used extensively in civil engineering field ${ }^{17}$. ANNs utilizing the supervised error Back-Propagation (BP) training algorithm ${ }^{18}$ are commonly referred to as BP neural networks. BP networks are the most popular ANN and have been used in this study. The structure of a single hidden layer BP network is shown in Fig. 2, where $\mathbf{x}$ and $\mathbf{0}$ are the input and 
output vectors respectively. The so-called bias inputs into the hidden and output layers have been represented by solid squares and both have the value of +1 . The weights for the whole network, denoted by vector $\mathbf{w}$, store information as in the brain and are learnt during the training process.

The basic function of a single neuron in either the hidden or output layers is to calculate the weighted sum of all inputs $u$ :

$$
u=\mathbf{v}^{T} \mathbf{x}
$$

and compute the neuron output $y$ :

$$
y=f(u)
$$

where the weights vector for the single neuron has been denoted by $\mathbf{v}$ to avoid confusion with $\mathbf{w}$ that contains the weights for all neurons, and $f$ is the neuron's activation function. The total error $E$ in the network is a function of the weights and can be written as:

$$
E(\mathbf{w})=\frac{1}{2} \mathbf{e}(\mathbf{w})^{T} \mathbf{e}(\mathbf{w})
$$

where $\mathbf{e}(\mathbf{w})$ is an error vector defined by:

$$
\mathbf{e}(\mathbf{w})=\mathbf{d}-\mathbf{o}(\mathbf{w})
$$

and $\mathbf{d}$ is the vector of target values or desired network outputs. In the training phase, the network calculates the output for a given input and the error is propagated backwards from the output layer to the preceding layers according to the BP algorithm. In the original algorithm a gradient descent methods was used to alter the weights so that the error was minimized. In this study a modified algorithm was used in which the weights were changed according to the LevenbergMarquardt algorithm ${ }^{19}$, a quasi-Newton method developed specifically for the sum of errors squared error function. The algorithm's application to the original BP algorithm is described $\mathrm{in}^{20}$. Introducing the Jacobian matrix $\mathbf{J}$ defined as:

$$
\mathbf{J}(\mathbf{w})=\frac{\partial \mathbf{e}}{\partial \mathbf{w}}
$$

The new weights can be found through the application of the following iterative process:

$$
\mathbf{w}_{k+1}=\mathbf{w}_{k}-\left[\mathbf{J}_{k}^{T} \mathbf{J}_{k}+\lambda_{k} \mathbf{I}\right]^{-1} \mathbf{J}_{k}^{T} \mathbf{e}_{k}
$$

where subscript $k$ denotes the iteration step. The parameter $\lambda_{k}$ is a scalar that controls convergence properties. If $\lambda_{k}$ is equal to zero, the Levenberg-Marquardt algorithm becomes the Gauss-Newton method.

\section{Hidden layer neurons}

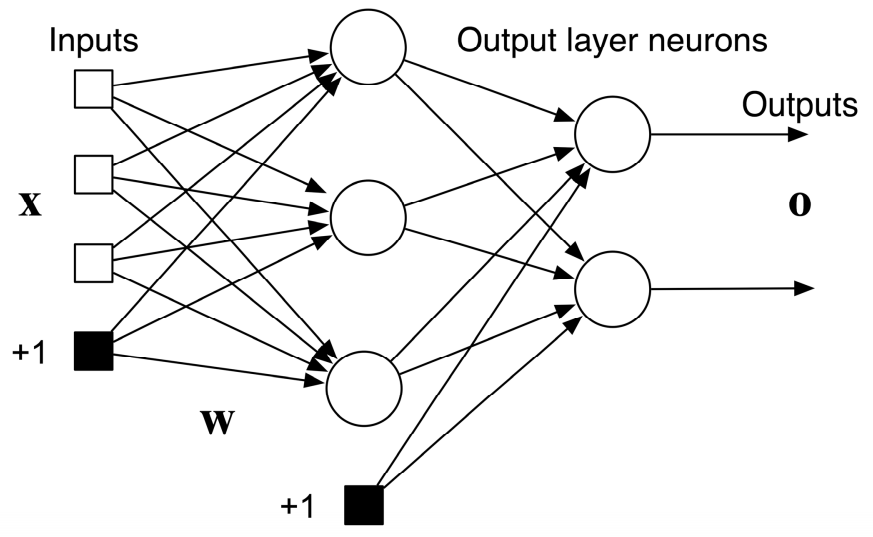

Fig. 2. A single hidden layer ANN. 


\section{Modal identification}

Although the proposed damage identification method does not use modal parameters as damage sensitive features, experimental modal analysis was conducted in order to obtain an insight into the system dynamics and to estimate modal parameters for updating of the computer models of the experimental structure in various damage states. The natural frequencies, viscous damping ratios and mode shapes of the test structure were determined from system identification of a discrete state-space model. A discrete state space model at time step $k$ can be written as follows:

$$
\begin{aligned}
& \mathbf{x}_{k+1}=\mathbf{A} \mathbf{x}_{k}+\mathbf{B} \mathbf{u}_{k} \\
& \mathbf{y}_{k}=\mathbf{C} \mathbf{x}_{k}+\mathbf{D} \mathbf{u}_{k}
\end{aligned}
$$

where $\mathbf{x}_{k}, \mathbf{u}_{k}$ and $\mathbf{y}_{k}$ are respectively the state, input and output vectors at time $k$, and $\mathbf{A}, \mathbf{B}, \mathbf{C}$ and $\mathbf{D}$ are system matrices to be determined in the identification process. In this case, the output vector was the three storey accelerations and the input vector was the table acceleration. The PEM identification algorithm implemented in the system identification toolbox in MATLAB ${ }^{21}$ was used to estimate the system matrices $\mathbf{A}, \mathbf{B}, \mathbf{C}$ and $\mathbf{D}$.

\section{Model updating}

In order to match experimental results, analytical modals can be updated on a range of experimental data including natural frequencies, mode shapes and frequency domain data ${ }^{22}$. Natural frequencies are often the most certain modal parameters obtained from modal analysis and are often used in the model updating process, as in this study. The error $\mathbf{e}_{i}$ between the analytical and experimental frequencies, $\omega_{a, i}$ and $\omega_{e, i}$, can be expressed as an error vector:

$$
\mathbf{e}_{i}=\frac{\omega_{a, i}^{2}-\omega_{e, i}^{2}}{\omega_{e, i}^{2}}
$$

where subscript $i$ refers to $i$-th mode. The analytical frequencies depend on a set of parameters, such as masses and member stiffnesses, denoted by vector $\theta$, that define the model. In the updating process, the vector of errors for all considered modes $\mathbf{e}$ is minimized by an iterative procedure where the updating parameters $\boldsymbol{\theta}_{k}$ at iteration step $k$ are adjusted using:

$$
\boldsymbol{\theta}_{k+1}=\boldsymbol{\theta}_{k}-\mathbf{S}_{k}^{+} \mathbf{e}_{k}
$$

where $\mathbf{S}^{+}$is the pseudoinverse of the sensitivity matrix whose entries can be evaluated as:

$$
\mathbf{S}_{i j}=\frac{1}{\omega_{a, i}^{2}} \boldsymbol{\phi}_{a, i}^{T}\left[\frac{\partial \mathbf{K}}{\partial \boldsymbol{\theta}_{j}}-\omega_{a, i}^{2} \frac{\partial \mathbf{M}}{\partial \boldsymbol{\theta}_{j}}\right] \phi_{a, i}
$$

where $\mathbf{K}$ and $\mathbf{M}$ are the stiffness and mass matrices and $\phi_{a, i}$ are the analytical mode shape vectors.

\section{TEST STRUCTURE AND DATA ACQUISITION}

The test structure used in this study was approximately $2.1 \mathrm{~m}$ high and constructed from equal angle aluminium column sections and stainless steel floor plates bolted together with aluminium brackets as shown in Fig. 1. The stainless steel plates were $4 \mathrm{~mm}$ thick and $650 \mathrm{~mm} \times 650 \mathrm{~mm}$ square. The column sections were $30 \mathrm{~mm} \times 30 \mathrm{~mm}$ equal angles. Two section thicknesses were used for the columns, either $3 \mathrm{~mm}$ or $4.5 \mathrm{~mm}$ for the damaged and undamaged states respectively. Each column was made of $3 \times 0.7 \mathrm{~m}$ high segments, rather than one long angle, in order to make them easily replaceable for simulation of localized damage at different stories. The column sections were fastened at each end with two M6 bolts to aluminium brackets. (Fig. 1b). Additional brackets were installed at the base of the structure to minimize torsional motion. The whole structure was mounted on a $20 \mathrm{~mm}$ plywood sheet bolted with M10 bolts to the shake table.

The structure was instrumented with four $2.5 \mathrm{Vg}^{-1}$ uniaxial accelerometers, one for measuring the table acceleration and one for each storey. Accelerations were measured in the direction of ground motion at $400 \mathrm{~Hz}$ using a computer fitted with a data-logging card. All data was filtered with a zero phase shift $50 \mathrm{~Hz}$ low pass filter. Afterwards the data was decimated by a factor of four for modal analysis and eight for time series modelling. This reduced the original $400 \mathrm{~Hz}$ 
signal down to $100 \mathrm{~Hz}$ and $50 \mathrm{~Hz}$ respectively. The decimate procedure implemented in MATLAB ${ }^{23}$ uses an eight order Chebyshev Type I lowpass filter with cutoff frequency $(0.8 / R) \times\left(F_{s} / 2\right)$, where $F_{s}$ is the initial sampling frequency and $R$ is the decimate factor, before resampling the data. The decimate process improved the quality of the data by further removing high frequency noise.

\section{OFFLINE DAMAGE DETECTION ON THE TEST STRUCTURE}

\section{Preliminary tasks: modal identification, model updating and time series analysis of accelerations}

The proposed offline method was applied to damage detection in the 3-storey test structure. Firstly, the modal parameters for the undamaged structure were estimated from five response records, containing four 10 s and one 20 s record. In all cases, the excitation or input was Gaussian white noise. Table 1 shows the estimated experimental natural frequencies $f_{e}$ and damping ratios $\xi$ obtained from the five intervals. The lower and upper bounds correspond to two standard deviations or $95 \%$ confidence levels. It can be seen that while natural frequencies show little error, the damping ratios were much more uncertain. The modes shapes were estimated from the 20 s record only. Table 2 gives the normalized mode shapes for a maximum value of 1.00 .

Damage was introduced into the structure by replacing all four columns at a particular storey (or stories) with thinner ones. Four damage states were considered; these were labelled D0, D1, D2 and D3 corresponded to no damage (i.e. healthy structure), $1^{\text {st }}$ storey damage, $2^{\text {nd }}$ storey damage, and simultaneous $1^{\text {st }}$ and $2^{\text {nd }}$ storey damage, respectively. Modal experiments and analyses, similar as for the healthy structure (damage state D0) were conduced and Table 3 lists the experimental frequencies $f_{e}$ in all four damage states. Table 3 also gives percentage changes of the frequencies $\Delta f_{e}$ in states D1, D2, and D3 in relation to D0.

In this study, the lateral stiffness of the structure in both the damaged and undamaged states could not be accurately determined from analytical investigations and accurate computer models set up. Initial experiments showed that the stiffness was much smaller than it would appear taken into account sectional properties of the columns and Young's modulus of aluminium. It was, however, observed that the structure lost it stiffness because of the way the column-floor joints were constructed (Fig. 1b). The use of relatively flexible mounting brackets combined with the lack of continuity of the column over the joint led to a considerable lateral stiffness loss. Precise analytical calculation of the stiffness of the joint was difficult. However, the knowledge of stiffness values is very useful when one wants to quantify experimental damage more accurately as a reduction in stiffness.

Table 1. Identified frequencies and damping ratios for healthy structure.

\begin{tabular}{ccc}
\hline Mode & $f_{e}(\mathrm{~Hz})$ & $\xi(\%)$ \\
\hline $1^{\text {st }}$ & $1.928 \pm 0.006$ & $0.6 \pm 0.2$ \\
$2^{\text {nd }}$ & $5.52 \pm 0.02$ & $0.6 \pm 0.2$ \\
$3^{\text {rd }}$ & $8.55 \pm 0.04$ & $0.8 \pm 0.4$ \\
\hline
\end{tabular}

Table 2. Normalized mode shapes for healthy structure.

\begin{tabular}{cccc}
\hline Storey & Mode 1 & Mode 2 & Mode 3 \\
\hline $1^{\text {st }}$ & 0.18 & 0.64 & 1.00 \\
$2^{\text {nd }}$ & 0.69 & 1.00 & -0.48 \\
$3^{\text {rd }}$ & 1.00 & -0.88 & 0.16 \\
\hline
\end{tabular}

Table 3. Natural frequencies and percentage changes at different damage states.

\begin{tabular}{cccccccc}
\hline & \multicolumn{3}{c}{$f_{e}(\mathrm{~Hz})$} & \multicolumn{3}{c}{$\Delta f_{e}(\%)^{\mathrm{a}}$} \\
\hline Mode & $\mathrm{D} 0$ & $\mathrm{D} 1$ & $\mathrm{D} 2$ & $\mathrm{D} 3$ & $\mathrm{D} 1$ & $\mathrm{D} 2$ & $\mathrm{D} 3$ \\
\hline $1^{\text {st }}$ & 1.928 & 1.879 & 1.837 & 1.840 & $-2.5 \%$ & $-4.7 \%$ & $-4.6 \%$ \\
$2^{\text {nd }}$ & 5.52 & 5.43 & 5.46 & 5.42 & $-1.6 \%$ & $-1.0 \%$ & $-1.8 \%$ \\
$3^{\text {rd }}$ & 8.55 & 8.30 & 8.09 & 8.15 & $-2.9 \%$ & $-5.3 \%$ & $-4.6 \%$ \\
\hline
\end{tabular}

${ }^{\mathrm{a}}$ Based on D0. 
For the purpose of experimental stiffness estimation, simple 3-storey lumped mass-spring analytical models were updated on the experimental natural frequencies obtained from the experimental modal analysis. The updating parameters were chosen to be the lateral stiffness of each storey $k_{1}, k_{2}$, and $k_{3}$. The initial stiffnesses were estimated by rough hand calculations giving $9300 \mathrm{~N} / \mathrm{m}$ for $k_{2}$ and $k_{3}$. Because of the additional brackets placed at the base of the structure, $k_{1}$ was expected be significantly greater than $k_{2}$ or $k_{3}$, and a value for $k_{1}$ of $40000 \mathrm{~N} / \mathrm{m}$ was used. Additionally, after the updating process was complete, the analytical mode shapes were checked against the experimental mode shapes using the popular Modal Assurance Criterion (MAC) ${ }^{22}$. Table 4 lists the results from the updated analytical models. The analytical frequencies $f_{a}$ are very close to their experimental counterparts reported previously in Table 3 . The MAC values show excellent correlation for the $1^{\text {st }}$ mode and the $2^{\text {nd }}$ and $3^{\text {rd }}$ modes show good correlation. The obtained storey stiffnesses are given in Table 5. Finally, Table 6 quantifies damage at each storey for all damage stases D0-D4 as the percentage of remaining stiffness.

For the purpose of simulating structural response, eight scaled earthquake records were used to excite the test structure on the shake table in the four damage states. Table 7 lists the earthquakes used, the peak ground acceleration (PGA) of the original and scaled records, the duration of the record and the frequency at which the earthquake was sampled. The earthquakes were scaled so that a range of response amplitudes was obtained, while ensuring no yielding of the structure occurred.

Table 4. Analytical frequencies and MAC values after updating.

\begin{tabular}{ccccccccc}
\hline & \multicolumn{2}{c}{ D0 } & \multicolumn{2}{c}{ D1 } & \multicolumn{2}{c}{ D2 } & \multicolumn{2}{c}{ D3 } \\
\hline Mode & $f_{a}(\mathrm{~Hz})$ & MAC & $f_{a}(\mathrm{~Hz})$ & MAC & $f_{a}(\mathrm{~Hz})$ & MAC & $f_{a}(\mathrm{~Hz})$ & MAC \\
\hline $1^{\text {st }}$ & 1.917 & 1.00 & 1.908 & 0.99 & 1.846 & 1.00 & 1.838 & 0.99 \\
$2^{\text {nd }}$ & 5.49 & 0.93 & 5.47 & 0.93 & 5.46 & 0.91 & 5.45 & 0.96 \\
$3^{\text {rd }}$ & 8.54 & 0.93 & 8.28 & 0.93 & 8.46 & 0.90 & 8.20 & 0.91 \\
\hline
\end{tabular}

Table 5. Updated stiffnesses from analytical models.

\begin{tabular}{cccccc}
\hline Stiffness & Initial estimate & D0 & D1 & D2 & D3 \\
\hline$k_{l}(\mathrm{~N} / \mathrm{m})$ & $4.00 \times 10^{4}$ & $3.77 \times 10^{4}$ & $3.49 \times 10^{4}$ & $3.77 \times 10^{4}$ & $3.49 \times 10^{4}$ \\
$k_{2}(\mathrm{~N} / \mathrm{m})$ & $0.93 \times 10^{4}$ & $0.60 \times 10^{4}$ & $0.60 \times 10^{4}$ & $0.54 \times 10^{4}$ & $0.54 \times 10^{4}$ \\
$k_{3}(\mathrm{~N} / \mathrm{m})$ & $0.93 \times 10^{4}$ & $0.77 \times 10^{4}$ & $0.77 \times 10^{4}$ & $0.77 \times 10^{4}$ & $0.77 \times 10^{4}$ \\
\hline
\end{tabular}

Table 6. Damage at each storey as the percentage of remaining stiffness.

\begin{tabular}{ccccc}
\hline & \multicolumn{4}{c}{ Percentage of remaining stiffness (\%) } \\
\hline & D0 & D1 & D2 & D3 \\
\hline $1^{\text {st }}$ storey & 1.00 & 0.93 & 1.00 & 0.93 \\
$2^{\text {nd }}$ storey & 1.00 & 1.00 & 0.90 & 0.90 \\
$3^{\text {rd }}$ storey & 1.00 & 1.00 & 1.00 & 1.00 \\
\hline
\end{tabular}

Table 7. Earthquake records used in the experiment.

\begin{tabular}{ccccc}
\hline Earthquake & PGA $(\mathrm{g})$ & Scaled PGA $(\mathrm{g})$ & Duration $(\mathrm{sec})$ & Sampling frequency $(\mathrm{Hz})$ \\
\hline Duzce 12/11/1999 & 0.535 & 0.027 & 25.885 & 200 \\
Erzincan 13/3/1992 & 0.496 & 0.033 & 20.780 & 200 \\
Gazli 17/5/1976 & 0.718 & 0.048 & 16.265 & 200 \\
Helena 31/10/1935 & 0.173 & 0.035 & 40.000 & 100 \\
Imperial Valley 19/5/1940 & 0.313 & 0.031 & 40.000 & 100 \\
Kobe 16/1/1995 & 0.345 & 0.035 & 40.960 & 100 \\
Loma Prieta 18/10/1989 & 0.472 & 0.047 & 39.945 & 200 \\
Northridge 1/17/1994 & 0.568 & 0.038 & 40.000 & 50 \\
\hline
\end{tabular}


The acceleration time history of each storey was modelled using a univariate AR model. A univariate AR(12) model was determined to give both a sufficient fit to the acceleration data and no significant correlation in the residual errors. The AR coefficients were estimated from a 500-point window advancing 100 points until the end of the record was reached. The least-squares approach described before was used to calculate the AR coefficients. A data set of 388 points containing 97 points for each damage state was obtained and randomly divided into 300 points for training and 88 points for testing the ANNs. As an example, Fig. 3 shows the statistical distribution (histogram) of the $1^{\text {st }}$ AR coefficient from the $1^{\text {st }}$ storey in the D0 and D1 damage states. To the naked eye, both distributions appear to be bi-modal with noticeable changes between them. However, precise detection of damage as well as extraction of further information e.g. the location or extent of damage, will require more robust and reliable tools such as an ANN.

\section{Simple damage classification}

Initially, an ANN was trained to distinguish between the four damage states only. The damage states D0, D1, D2 and D3

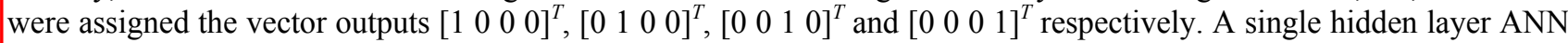
with 5 hidden layer neurons was found to give excellent results with $100 \%$ correct classification.

\section{Damage quantification and localization}

In this section, the ANNs were trained to relate the AR coefficients to the remaining stiffness at each storey, providing more useful information about the extent and location of damage. The damage at each storey, listed in Table 6, was defined as the current stiffness divided by the undamaged stiffness.

A single hidden layer ANN with 5 hidden layer neurons was found to give good predictions. The results have been shown graphically in Fig. 4, where the detected damage has been plotted against the actual damage for all three stories. For perfect predictions, the data points should lie on $(0.93,0.93)$ and $(1.00,1.00)$ for the $1^{\text {st }}$ storey, $(0.90,0.90)$ and $(1.00,1.00)$ for the $2^{\text {nd }}$ storey and $(1.00,1.00)$ for the $3^{\text {rd }}$ storey. The figure shows that the ANN has correctly detected the damage at each story with a small amount of scatter about the actual damage.

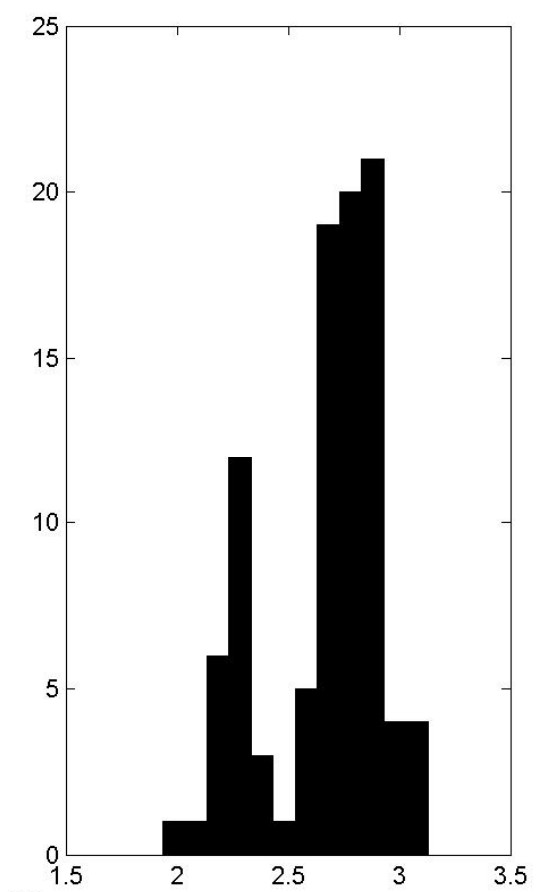

(a)

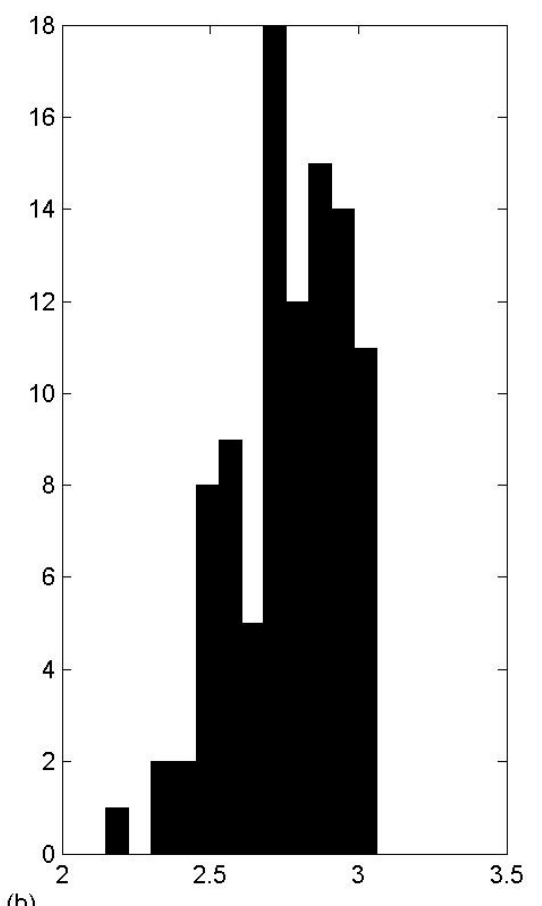

(b)

Figure 3. Histograms of the $1^{\text {st }}$ AR coefficient from the $1^{\text {st }}$ storey: (a) damage state D0, and (b) damage state D1. 

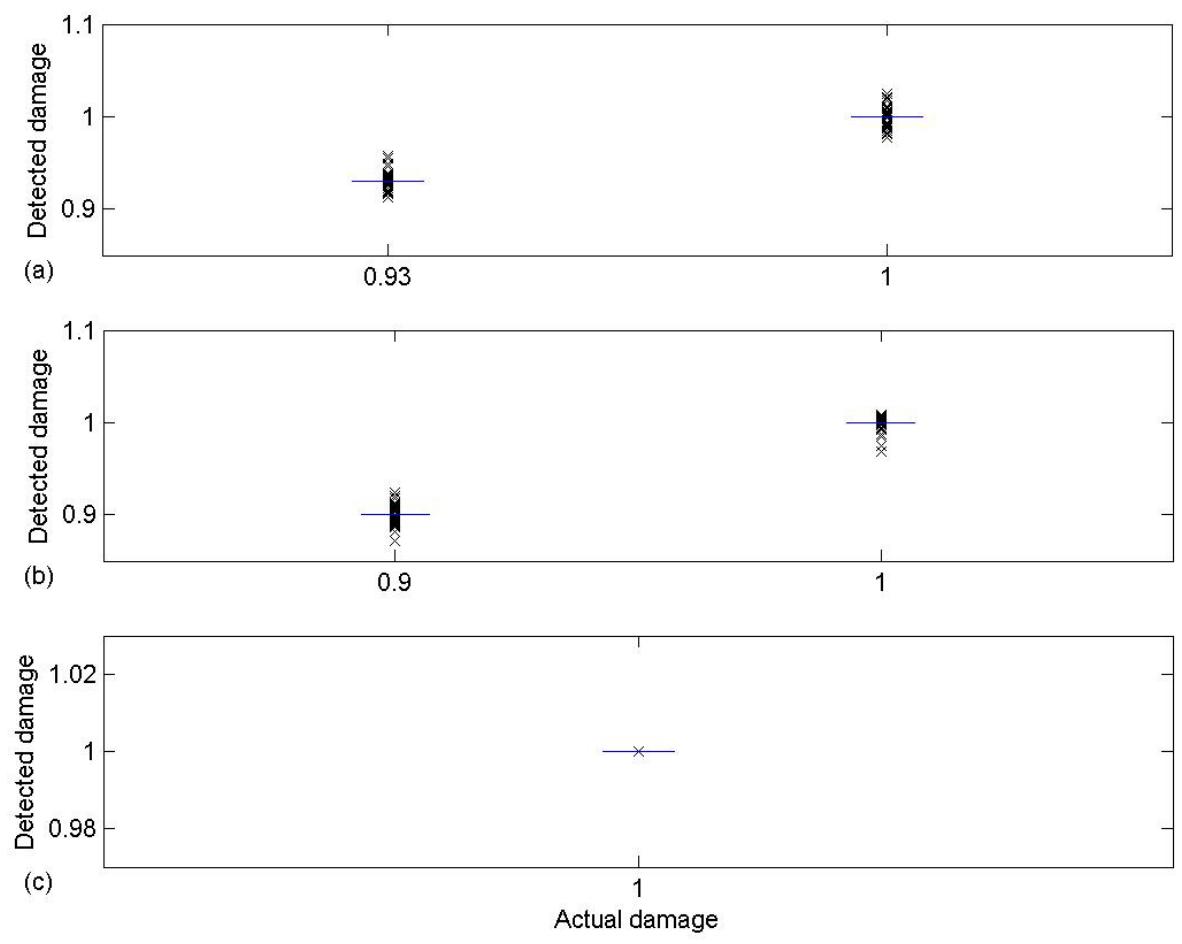

Figure 4. Detected vs. actual damage (a) $1^{\text {st }}$ storey, (b) $2^{\text {nd }}$ storey, and (c) $3^{\text {rd }}$ storey.

\section{NUMERICAL SIMULATIONS OF ONLINE DAMAGE DETECTION METHOD}

This section presents preliminary numerical results of the online damage detection method. The approach proposed is as follows. The training of an ANN to recognize changes in stiffness is performed offline where the input comprises ARX coefficients of the acceleration time series also identified online. However, once the ANN has been trained the system is subjected to a ground motion and unknown gradual stiffness degradation, the ARX coefficients are identified recursively using a Kalman filter, and the ANN is expected to trace stiffness loss as it progresses with time. The use of ARX models rather than previously employed pure AR models was motivated by the results of preliminary simulations which showed a rather poor performance of the AR models for the online method.

The proposed online scheme was applied to a single degree of freedom (SDOF) system. The system had a mass of $m=1 \times 10^{4} \mathrm{~kg}$, a lateral stiffness of $k=1 \times 10^{6} \mathrm{~N} / \mathrm{m}$ and a viscous damping ratio of $\xi=0.05$. This gave a natural period of 0.63s. For the purpose of ANN training, four levels of damage were investigated; 1 (no damage), 0.8, 0.6 and 0.4 times the original stiffness. The SDOF system was subjected to excitation by white noise and 1000-point response time histories were fitted using an $\operatorname{ARX}(2,4)$ model with a time delay $k$ of $1.5 \%$ Gaussian noise was added to the time histories. 200 samples of ARX coefficients were calculated for each damage state using the offline least-squares method. The 800-point data set was randomly divided into 600 points for ANN training and the remaining 200 points were used to test the ANN. The ANN was trained to relate the ARX coefficients to the reduction in stiffness.

To test the ANN and detect damage on online data, the SDOF system was simulated with a linear reduction in stiffness over a 200-point interval in the middle of a 6000-point response. Again, 5\% Gaussian noise was added to the time history. The ARX coefficients were calculated using the Kalman filter and fed into the ANN, trained previously, to give an online estimate of damage. In the example, a 0.8 reduction in stiffness was simulated. Figure 1 shows the detected damage closely tracks the simulated value after some initial lag at the onset of damage.

The results showed that ARX models and the Kalman filter were excellent tools for tracking changes in a SDOF system over time. Incorporated with an ANN, this information was able to be successfully interpreted as damage. 


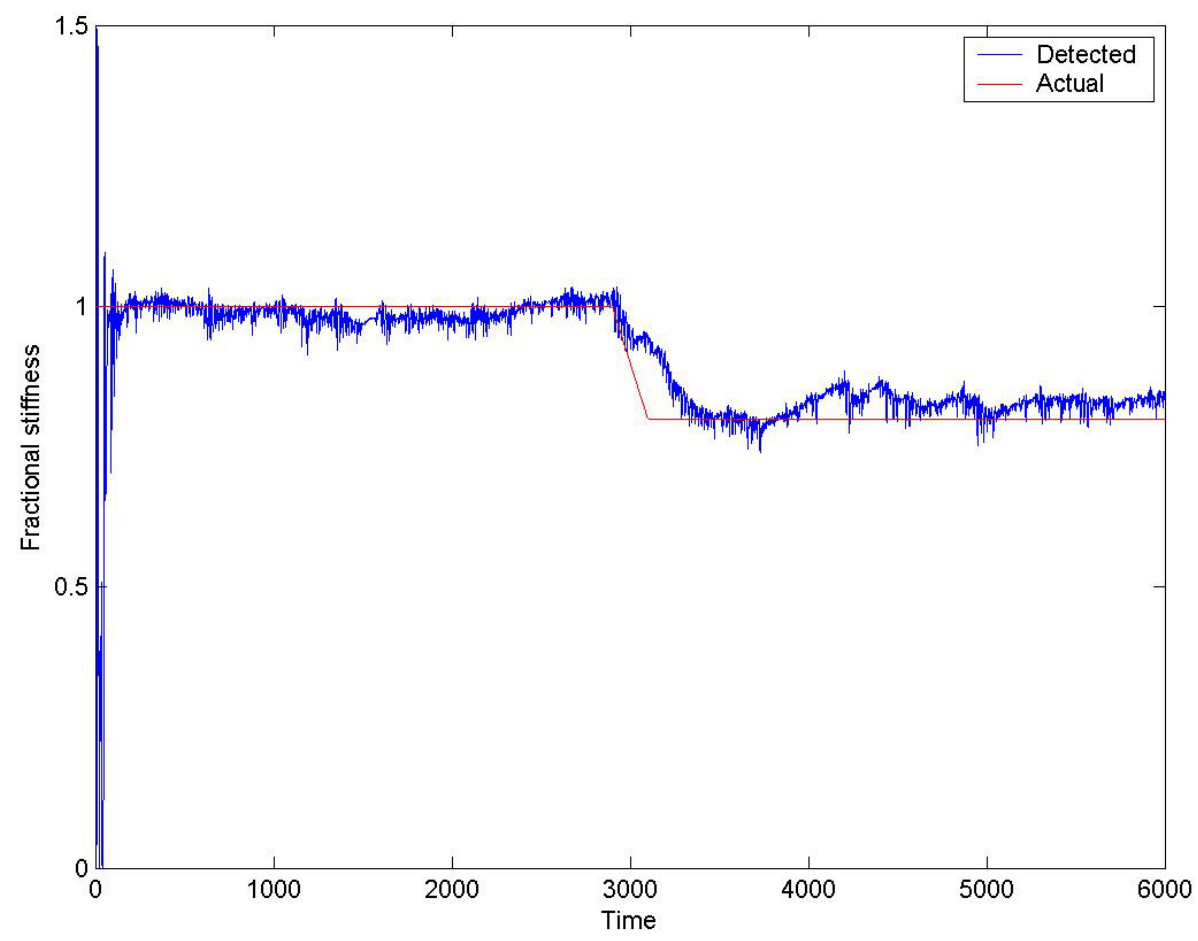

Figure 5. Online damage detection results.

\section{CONCLUSIONS}

In this study, a damage detection and localization method using AR models and ANNs has been developed. The method firstly identifies AR or ARX models for acceleration time histories, uses the coefficients of these models as damage sensitive features, and feeds them into an ANN to build a relationship between them and the remaining stiffness of the damaged structure. An offline variant of the method has been applied to a 3-storey laboratory structure with various damage scenarios simulated by stiffness reduction. Acceleration time histories of the structure under earthquake motion were recorded on a shake table. An online version where time series coefficients and damage are identified recursively has been preliminarily investigated through numerical simulations. The proposed method has been demonstrated to be able to classify and detect and track damage with very good accuracy.

\section{Acknowledgement}

The authors would like to express their gratitude for the Earthquake Commission Research Foundation of New Zealand for their financial support of this research (Project No. UNI/535).

\section{REFERENCES}

1. S. W. Doebling, C. R. Farrar, M. B. Prime and D. W. Shevitz, Damage identification and health monitoring of structural and mechanical systems from changes in their vibration characteristics: A literature review, LA13070-MS, Los Alamos National Laboratory, Los Alamos, New Mexico, 1996. 
2. H. Sohn, C. R. Farrar, F. M. Hemez, D. D. Shunk, D. W. Stinemates and B. R. Nadler, A review of structural health monitoring literature: 1996-2001, LA-13976-MS, Los Alamos National Laboratory, Los Alamos, New Mexico, 2003.

3. H. Sohn, J. A. Czarnecki and C. R. Farrar, "Structural health monitoring using statistical process control," Journal of Structural Engineering 126(11), 1356-1363 (2000).

4. H. Sohn, C. R. Farrar, N. F. Hunter and K. Worden, Applying the lanl statistical pattern recognition paradign for structural health monitoring to data from a surface-effect fast patrol boat, LA-13761-MS, Los Alamos National Laboratory, Los Alamos, 2001.

5. P. Omenzetter and J. M. W. Brownjohn, "Application of time series analysis for bridge monitoring," Smart Materials and Structures 15(1), 129-138 (2006).

6. K. K. Nair, A. S. Kiremidjian and K. H. Law, "Time series-based damage detection and localization algorithm with application to the asce benchmark structure," Journal of Sound and Vibration 291(1-2), 349-368 (2006).

7. $\quad$ F. Casciati and S. Casciati, "Structural health monitoring by lyapunov exponents of non-linear time series," Structural Control and Health Monitoring 13(1), 132-146 (2006).

8. J. M. Nichols, L. N. Virgin, M. D. Todd and J. D. Nichols, "On the use of attractor dimension as a feature in structural health monitoring," Mechanical Systems and Signal Processing 17(6), 1305-1320 (2003).

9. J. M. Nichols, M. Seaver and S. T. Trickey, "A method for detecting damage-induced nonlinearities in structures using information theory," Journal of Sound and Vibration 297(1-2), 1-16 (2006).

10. K. Worden, G. Manson and N. R. J. Fieller, "Damage detection using outlier analysis," Journal of Sound and Vibration 229(3), 647-667 (2000).

11. Y. Q. Ni, X. T. Zhou and J. M. Ko, "Experimental investigation of seismic damage identification using pcacompressed frequency response functions and neural networks," Journal of Sound and Vibration 290(1-2), 242263 (2006).

12. C. Zang and M. Imregun, "Structural damage detection using artificial neural networks and measured frf data reduced via principal component projection," Journal of Sound and Vibration 242(5), 813-827 (2001).

13. M. Nakamura, S. F. Masri, A. G. Chassiakos and A. K. Caughey, "A method for non-parametric damage detection through the use of neural networks," Earthquake Engineering and Structural Dynamics 27(997-1010 (1998).

14. W. W. S. Wei, Time series analysis: Univariate and multivariate methods, 2 ed, Pearson, Boston, 2006.

15. L. Ljung and T. Soderstrom, Theory and practice of recursive identification, MIT Press, Cambridge, Massachusetts, 1983.

16. A. C. Harvey, Forecasting, structural time series models and the kalman filter, Cambridge University Press, New York, 1989.

17. H. Adeli, "Neural networks in civil engineering: 1989-2000," Computer-Aided Civil and Infrastructure Engineering 16(2), 126-142 (2001).

18. D. E. Rumelhart and J. L. McClelland, Parallel distributed processing: Explorations in the microstructure of cognition, MIT Press, Cambridge, MA., 1986.

19. D. Marquardt, "An algorithm for least squares estimation of nonlinear parameters," Journal of the Society for Industrial and Applied Mathematics 11(2), 431-441 (1963).

20. M. T. Hagan and M. Menhaj, "Training feedforward networks with the marquardt algorithm," IEEE Transactions on Neural Networks 5(6), 989-993 (1994).

21. L. Ljung, System identification toolbox: User's guide version 6, Mathworks, Natick, MA, 2006.

22. M. I. Friswell and J. E. Mottershead, Finite element model updating in structural dynamics, Kluwer Academic Publishers, Dordrecht, 1995.

23. Signal processing toolbox: User's guide version 6, Mathworks, Natick, MA, 2006. 